\title{
GROUND-BASED OBSERVATIONS OF TYPE III BURSTS
}

\author{
R. T. STEWA RT \\ Division of Radiophysics, CSIRO, Sydney, Australia
}

\begin{abstract}
Observations over the past 20 yrs or so are reviewed, with emphasis on recent high spatial resolution observations. The results lend support to earlier ideas on the propagation of type III electron streams through coronal regions of weak magnetic field strength but have not as yet settled the question whether the electrons propagate along the axes of coronal streamers. Several important burst properties appear to be significantly affected by ray scattering on small-scale size density irregularities in the corona.
\end{abstract}

\section{Introduction}

The type III burst was first recognized as a distinct class of solar radio emission from spectral studies at metre wavelengths (Wild, 1950). During the past two decades the type III burst has been studied in great detail with groundbased spectrographs, interferometers and heliographs, and also with satellite-based spectrographs. The bursts have been observed at frequencies as high as $\sim 600 \mathrm{MHz}$ and as low as $30 \mathrm{kHz}$, corresponding to source heights near the base of the corona and the Earth's orbit respectively. The radio emission has been shown to be the result of plasma waves excited by a stream of fast electrons ejected from near the region of a chromospheric flare and travelling outwards, sometimes as far as the Earth's orbit. Since the electrons escape along magnetic field lines they act as tracers of the coronal and inter-planetary magnetic fields. The source heights at various frequencies give information about the electron density distribution along the electron path. The exponential decay rates of the burst at different frequencies have been used to estimate the electron temperatures in the corona. These and other aspects of the type III burst will be discussed in the light of recent ground-based observations. For earlier reviews of ground-based observations of type III bursts see Wild et al. (1963), Kundu (1965), Wild and Smerd (1972), and Solar Radio Group Utrecht (1974).

\section{Burst Characteristics}

Type III bursts are a common form of sporadic radio emission, characterized by rapid frequency drift from high to low frequencies and by short duration. They occur as isolated bursts (as members of a connected group), sometimes regularly spaced (Figure 1a), and in very large numbers as members of a persistent storm. Isolated bursts often accompany the flash phase of a chromospheric flare (Loughead et al., 1957; Malville, 1962). Storms usually follow a large flare and can last several days. During the storm, type I bursts occur at higher frequencies $(>100 \mathrm{MHz})$ than type III bursts, which at lower frequencies may blend into a 'decametric continuum' (de la Noë et al., 1973).

Bursts are rich in variety. At one extreme is the short-duration type III burst with 
a rapid drift from high to low frequencies (Figure 1a). At the other extreme is the long-duration type III-V burst lasting $\simeq 1 \mathrm{~min}$ at metre wavelengths (Figure $1 \mathrm{c}$ ). In between are intermediate-duration bursts, which may be a mixture of type III and type V (Figure $1 \mathrm{~b}$ ). The starting frequency varies from burst to burst and can be as high as $600 \mathrm{MHz}$ or as low as $25 \mathrm{MHz}$. Sometimes bursts have both fundamental and second-harmonic components, which are difficult to distinguish because of the rapid drift rate (Figure 1b). Some bursts bend over at low frequencies and are known as 'inverted- $U$ ' bursts (Figure 1d). Another sub-type, the type IIIb burst, will be described

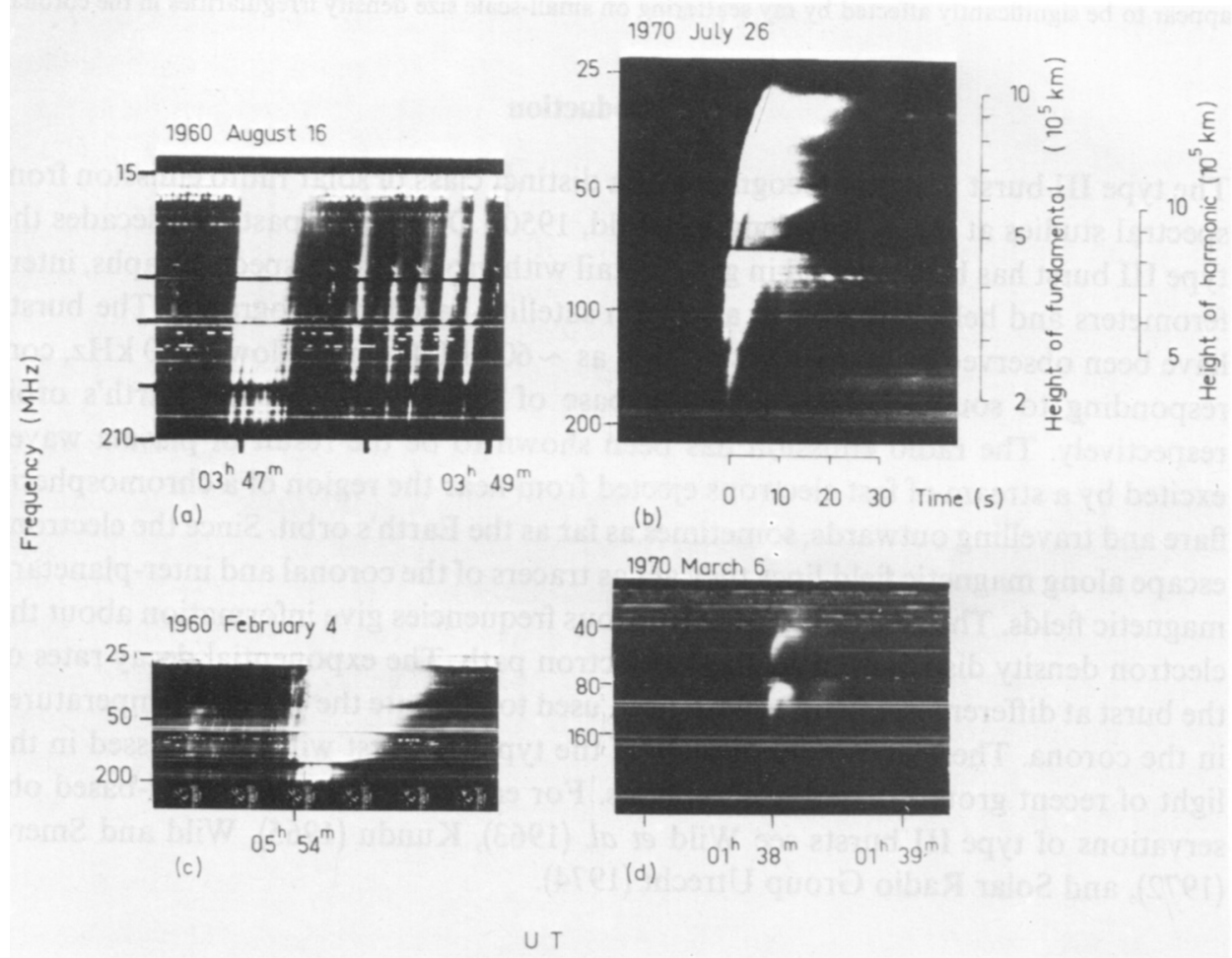

Fig. 1. (a) Groups of regularly spaced type III bursts (Wild et al., 1963). (b) A type III burst showing fundamental and second-harmonic structure followed by brief type $V$ continuum (Wild and Smerd, 1972). (c) An intense type III-V burst (Weiss and Stewart, 1965). (d) An inverted-U burst showing fundamental and second harmonic structure.

in Section 11. From the ground, the lowest observing frequency (determined by the ionospheric cut-off) is $\gtrsim 7 \mathrm{MHz}$.

At any frequency the intensity profile of a type III burst shows a rapid rise followed by an exponential decay. The decay rate decreases with decreasing frequency and so the duration increases. Decay rates, if due to collisional damping, give an upper limit to electron temperature in the corona. (See Section 10 for discussion on the effects of scattering of the radiation on coronal irregularities.) 


\section{Source Height}

According to the plasma hypothesis (Wild, 1950) type III emission occurs at the plasma frequency $f_{p}=9 \times 10^{-3} N^{1 / 2} \mathrm{MHz}$, where $N \mathrm{~cm}^{-3}$ is the electron density. The frequency drift results from the outward movement of the source through regions of decreasing electron density, i.e. decreasing plasma frequency. Hence the frequency drift, which is most clearly defined by the leading edge of the burst, represents the outward velocity of the source; the more rapid the drift, the faster the source velocity.

The plasma hypothesis was confirmed by one-dimensional interferometer measurements of the type III source height at different frequencies which showed successively lower frequencies being emitted from successively greater heights (Wild $e t$ al., 1959). The observed heights of type III bursts (if assumed to be fundamentals) give coronal electron densities which are about four times the values derived by white-light observations of the quiet $K$-corona (Figure 2). Hence it has been assumed

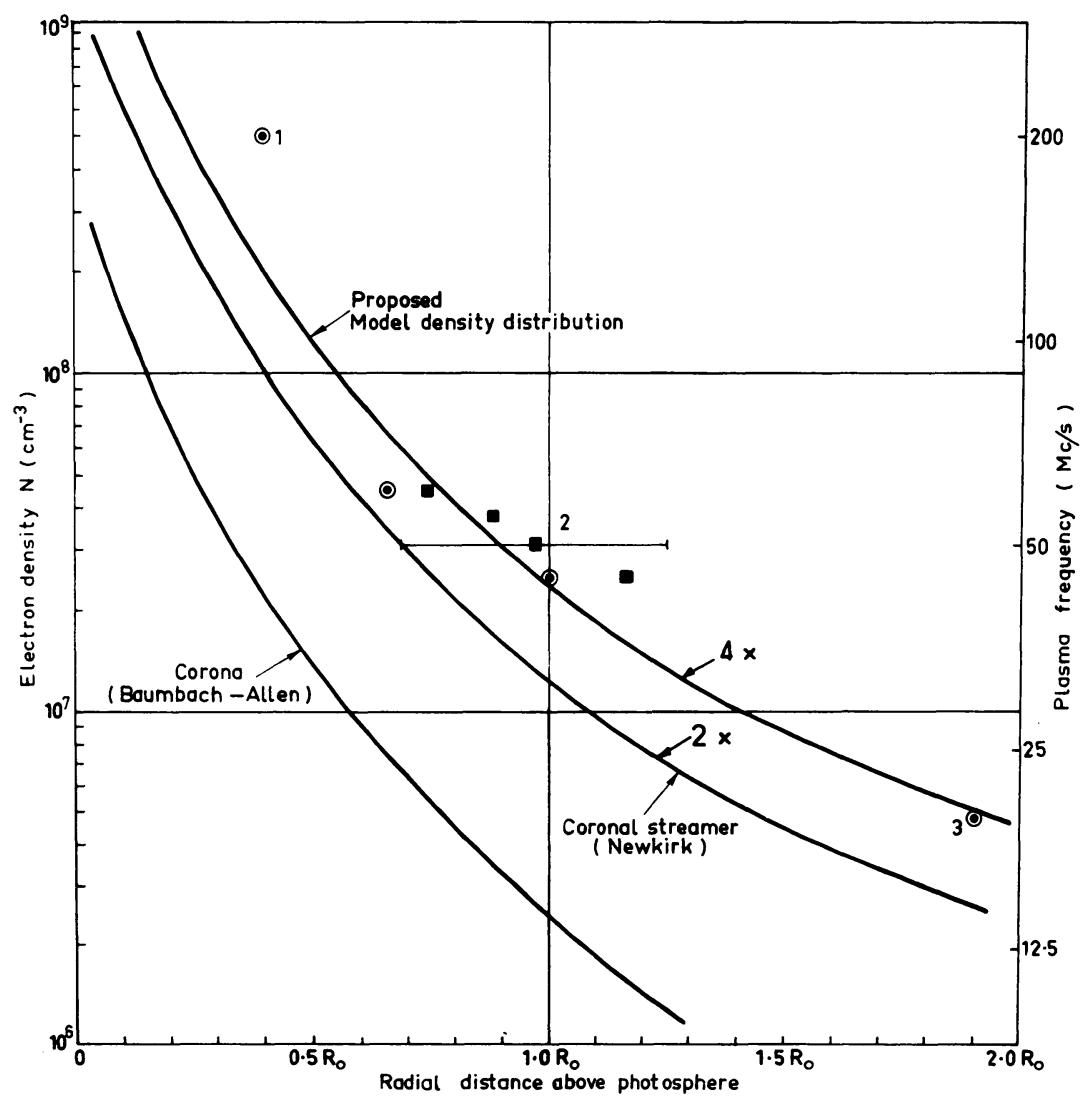

Fig. 2. A comparison of radio and optical determinations of electron density in the low corona. The plotted points give the heights of emission (fundamental) of type II and type III bursts measured by groundbased observers (Weiss, 1963). Note that Newkirk (1961) coronal streamer densities $=2 \times$ Newkirk (1961) quiet Sun densities (Wild et al., 1963). 
by many authors that the type III disturbance propagates along the axis of a coronal streamer, where the densities are higher than the surrounding corona. (See discussion on the effects of scattering in Section 8.)

\section{Source Velocity}

Interferometer observations show that the average radial velocity varies from 0.2 to $0.8 c$ (Figure 3) between the 60 and $45 \mathrm{MHz}$ plasma levels. Individual type III bursts observed over a frequency range from 200 to $12 \mathrm{MHz}$, i.e. from $0.15 R_{\odot}$ to $2.0 R_{\odot}$ above the photosphere, have drift rates which correspond to radial source velocities

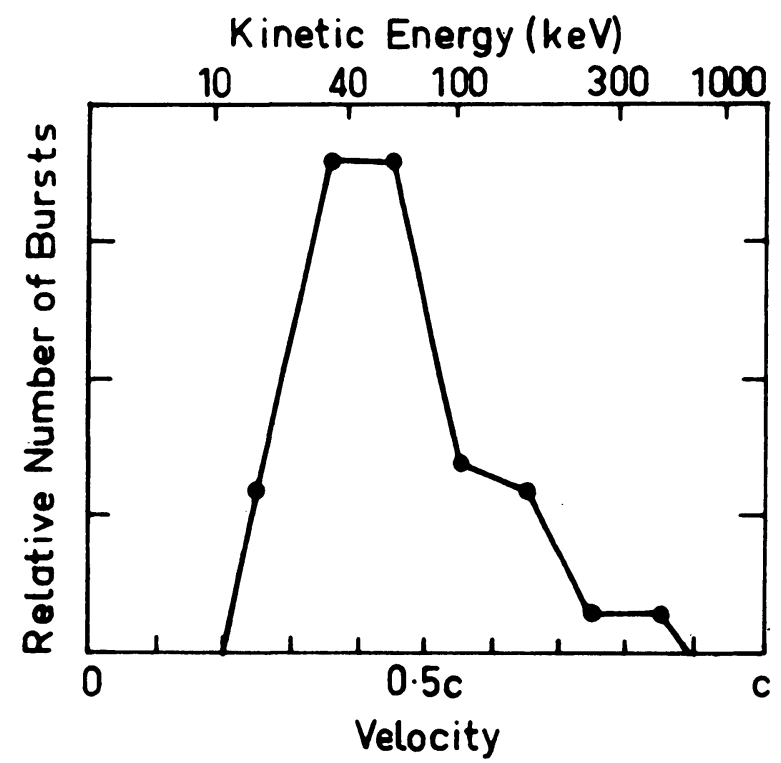

Fig. 3. Distribution of the speed of exciters of type III bursts assumed to be electrons traversing radial path (Wild and Smerd 1972, from velocity data of Wild et al., 1959).

$\sim c / 3$. The constancy of the radial component of the source velocity over such great distances suggests that the electrons in the type III disturbance travel without substantial spiralling along magnetic field lines (Stewart, 1965). Confirmation has come from satellite observations at hectometre wavelengths (Slysh, 1967; Fainberg and Stone, 1970; Haddock and Alvarez, 1973) which show that the average radial velocity of type III bursts is more or less constant, $\sim 0.3$ to $0.4 c$, out to heights $\sim 100 R_{\odot}$, although Fainberg et al. (1972) claim that some type III bursts do show a systematic deceleration with distance from the Sun. (See also review article by Fainberg (1974) in this issue.) The derived electron densities are in reasonable agreement with the density model based on metre wavelength observations if it is assumed that the observed radiation at low frequencies $(\leqslant 1 \mathrm{MHz})$ is second harmonic, as the results of Haddock and Alvarez (1973) suggest. Recent work by Alvarez and Haddock (1973) 
has shown that the observed frequency drift rates, for frequencies between $550 \mathrm{MHz}$ and $75 \mathrm{kHz}$, can be used to derive electron density models out to $1 \mathrm{AU}$ which are in good agreement with models based on optical and particle observations. (See Figures 3 and 5 of Alvarez and Haddock (1973).) Their theory assumes the exciter particles travel with constant velocity and zero pitch angle along an Archimedes spiral and ignores ray scattering and refraction effects.

\section{Electrons or Protons}

The question of whether the source particles are electrons or protons has been more or less settled by satellite observations of high fluxes of impulsive bursts of solar electrons at the Earth's orbit within 30 min following a flare and a type III burst (Van Allen and Krimigis, 1965; Anderson and Lin, 1966). Simultaneous observations of solar electrons and hectometric type III bursts from the Imp-6 space craft show that the onset of radio emission located at $1 \mathrm{AU}$ corresponds to the arrival of electrons of $\sim 100 \mathrm{keV}$ energy. The emission maximum corresponds to the arrival of slower $(\sim 10 \mathrm{keV})$ electrons (Lin et al., 1973). Hence the observed energy range of solar electrons 10 to $100 \mathrm{keV}$ is in good agreement with the speed of type III bursts observed at metre wavelengths (see Figure 3). Lin et al. (1973) also point out that the apparent deceleration of the type III drift rate with distance from the Sun may be due to pitch-angle scattering and not to energy loss. (See also review article by Lin (1974) in this issue.) Fluxes of energetic protons have been reported (McDonald and Van Hollebeke, 1973) but these fluxes are very low.

In addition, Kane (1972) has shown that impulsive hard X-ray bursts often occur almost simultaneously with type III bursts which extend into the decimetre band (Figure 4). The X-ray bursts are attributed to bremsstrahlung from non-thermal electrons located in the lower corona $\left(<0.1 R_{\odot}\right.$ above the photosphere). The derived electron energy is in the range 10 to $100 \mathrm{keV}$, i.e. similar to that of type III bursts (Kane, 1972). (See also review paper by Kane (1974) in this issue.)

\section{Origin of Type III Electrons}

Statistical studies by various authors (Loughead et al., 1957; Swarup et al., 1960; Malville, 1962) have shown that the correlation of isolated type III bursts (as distinct from storm bursts) with flares is quite high. Recent high-resolutions $\mathrm{H} \alpha$ studies (Kuiper and Pasachoff, 1973; Vorphal and Zirin, 1972) show that the occurrence of type III bursts is often closely related in time with $\mathbf{H} \alpha$ brightenings, which are also associated with X-ray and microwave bursts (see also Kane, 1972). Martres et al. (1972) and Axisa et al. (1973) find that some type III bursts occur associated closely in time $( \pm 1 \mathrm{~min})$ with the appearance of $\mathrm{H} \alpha$ absorbing features even when there is no detectable flare brightening. These features occur at the border of active regions along a line separating opposite sense of the longitudinal photospheric magnetic field $\left(H_{\|}=0\right)$. Axisa et al. (1973) suggest that the transient disturbance which causes 


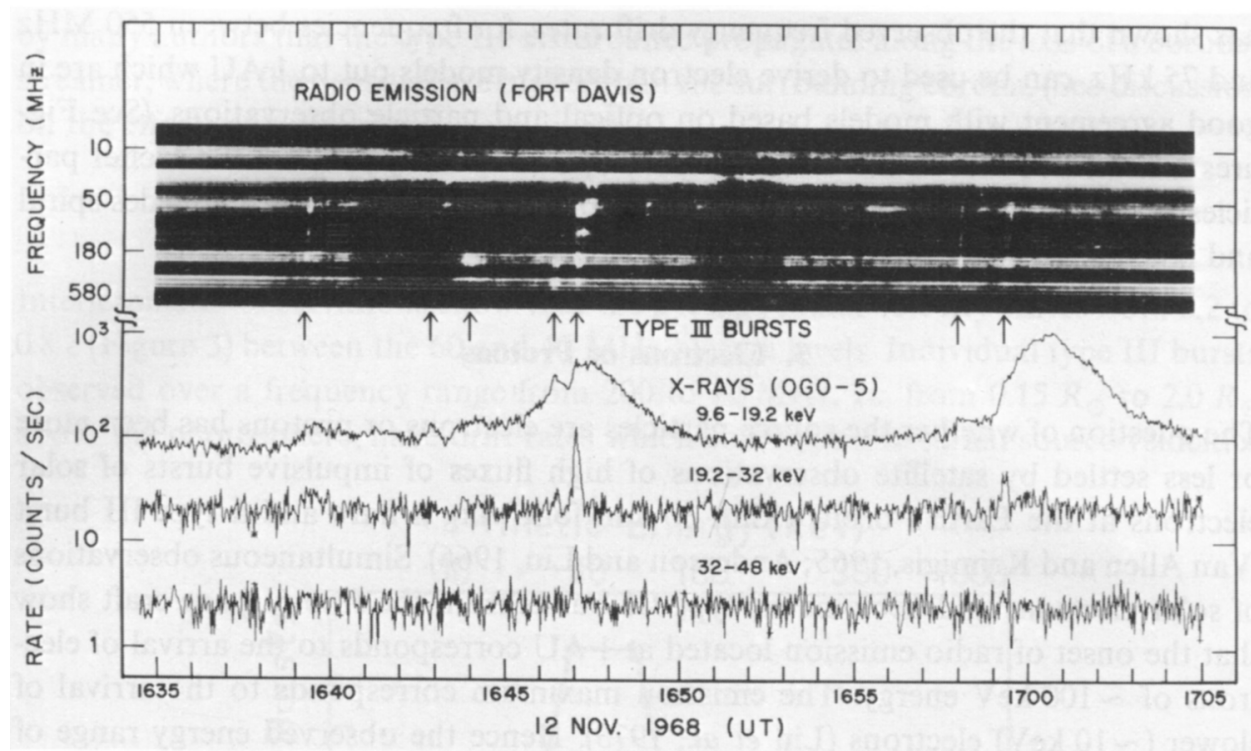

Fig. 4. An example of time-correlation between impulsive solar $\mathrm{X}$-rays $\geqslant 10 \mathrm{keV}$ and type III solar radio bursts in the 10 to $580 \mathrm{MHz}$ frequency range. Note the large spike at 1647 UT (Kane, 1972).

this chromospheric feature may also at times trigger the type III emission and the $\mathrm{H} \alpha$ flare. Teske et al. (1971) report several type III bursts occurring in the absence of flares (perhaps associated with $\mathrm{H} \alpha$ absorbing features (Kane et al., 1974)) but preceded by weak soft X-ray bursts, an observation which suggests that a thermal event precedes the type III instability.

However, the starting frequencies of type III storm bursts and also of some isolated type III bursts can be less than $100 \mathrm{MHz}$. If the type III electrons are always accelerated in the lower corona, say $\leqslant 0.1 R_{\odot}$ above the photosphere, one wonders why the starting frequencies of the type III bursts are often so small. Do the electrons travel over considerable distances in the lower corona without emitting radiation, or are the electrons accelerated locally near their starting frequency?

\section{Role of the Coronal Magnetic Field}

Wild and Smerd (1972) have summarized published observations and ideas on type III bursts in the model of Figure 5.

Electron acceleration and injection are assumed to occur in an unstable region containing opposing magnetic lines of force. Electrons which have access to open field lines around neutral planes (supposed to occur along the axis of the coronal streamer) give rise to type III bursts, while others injected into closed loop structures produce either $U$ bursts if the electrons are guided only once around the loop and then dispersed, or type $\mathrm{V}$ bursts if the electrons are trapped for periods $\dot{\sim} 1 \mathrm{~min}$.

Supporting evidence for the propagation of electrons in a weak field region has 
come from observations of a low degree of circular polarization* in type III bursts (Kai, 1970; Chernov et al., 1972). (See Section 9 for a discussion of linear polarization measurements.) Kai (1970) estimates the field strength in the type III source to be $<0.14 \mathrm{G}$ while Melrose and Sy (1972) estimate it to be $<0.04 \mathrm{G}$ (see also Rosenberg, 1973). Melrose and Sy base their estimates on observations of a smaller degree of

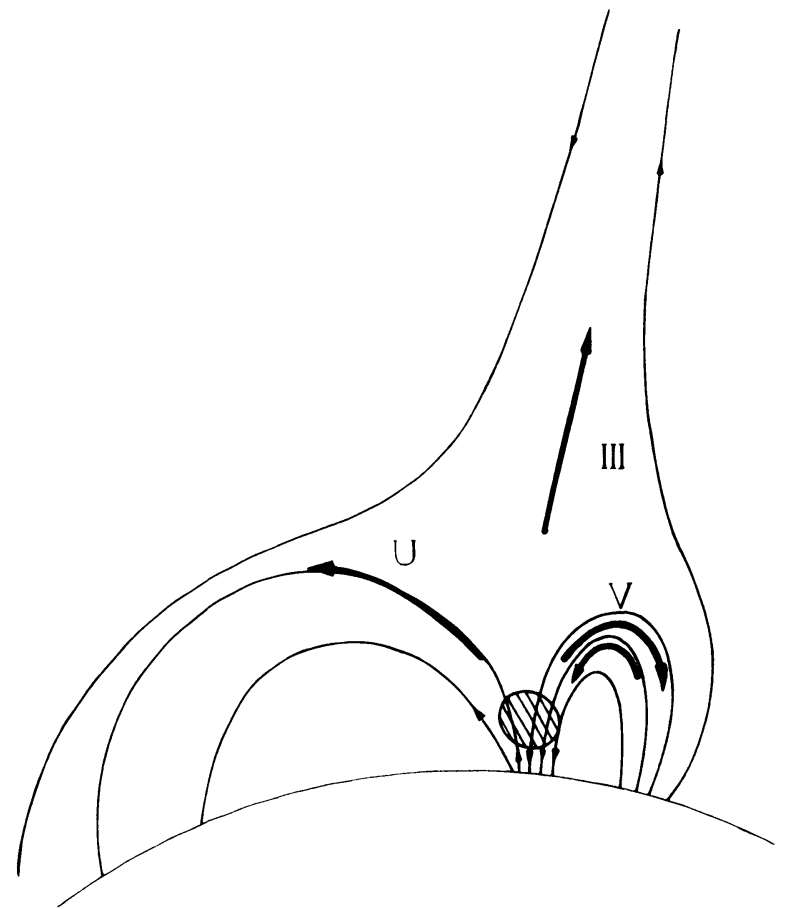

Fig. 5. Schematic magnetic field configuration to illustrate the possible different paths taken by electrons, ejected in the flash-phase explosion (hatched region) in classical type III, type $\mathrm{V}$ and inverted- $U$ bursts (Wild and Smerd, 1972).

circular polarization in harmonics than in.fundamental (McLean, 1971). Kai's observation of bipolar structure in type I bursts at $80 \mathrm{MHz}$ is a strong argument for the source of the type I bursts being in a closed field region above a sunspot group (Figure 6a). His observation of a displacement between associated type I and type III bursts is attributed to the propagation of type III electrons along open field lines away from the sunspot region (Figure 6b). For storms, displacements between type I and type III burst centres have also been observed (Stewart and Labrum, 1972; see also Figure 9).

Several authors (Weiss and Wild, 1964; Gleeson, 1965) have shown that if a group of electrons are injected isotropically into a diverging neutral sheet region then they will be guided outwards along the neutral plane. Evidence for the possible channelling

* An exceptionally high degree of circular polarization has been measured at the beginning of some type III bursts (Slottje, 1974; Rao, 1965). 
of type III bursts along a neutral plane has been reported by McLean (1970): at 80 $\mathrm{MHz}$ the members of a type III group have been observed to be strung out along a narrow segment of the solar disk (Figure 7). Position shifts between the leading and trailing arms of $U$ bursts at $80 \mathrm{MHz}$ have been observed (Labrum and Stewart, 1970). This result is consistent with the model of Figure 5. Position shifts have also been
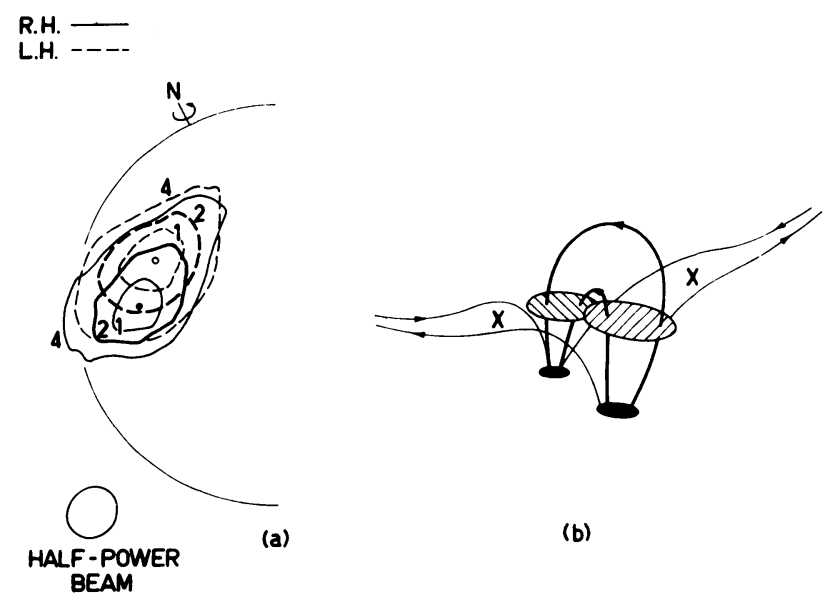

Fig. 6. (a) $80 \mathrm{MHz}$ contours of a type I storm source in the two senses of circular polarization showing bipolar structure. Contours marked 1,2 and 4 signify intensities $1 / \sqrt{ } 2,1 / 2$ and $1 / 4$ of the peak total (RH + LH) value (Wild, 1970, after Kai, 1970). (b) Kai's (1970) model of a bipolar storm centre. Type I emission originates from the shaded regions where strong magnetic fields intersect the plasma level. Type III emission originates from weak-field or neutral regions, such as those marked $x$

(Wild, 1970, after Kai, 1970).

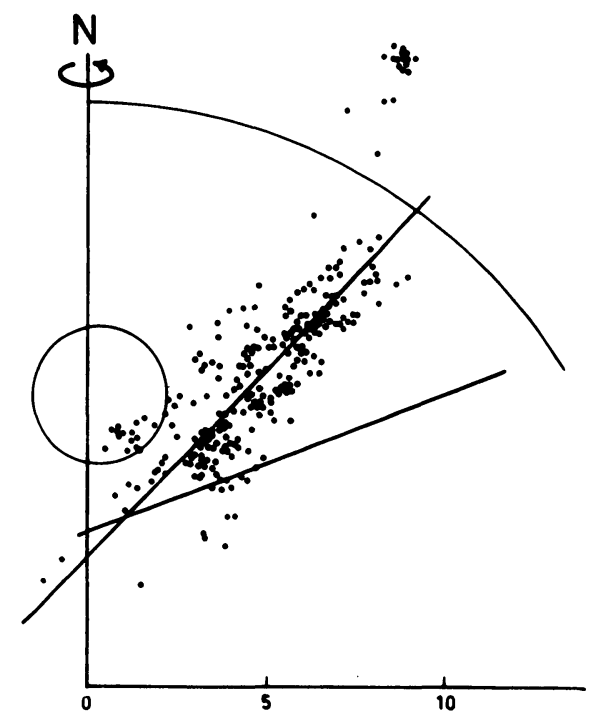

Fig. 7. The $80 \mathrm{MHz}$ radioheliograph centroids of burst sources, for each second of available data from 1968 November $13,00^{\mathrm{h}} 18^{\mathrm{m}} 32^{\mathrm{s}}$ to $00^{\mathrm{h}} 36^{\mathrm{m}} 00^{\mathrm{s}}$. The lower line and the circle show the scatter of points for earlier phases (McLean, 1970). 
observed between type $\mathrm{V}$ bursts and associated type III bursts (Labrum, unpublished; Kundu et al., 1970), while the type V source is considerably larger than the type III (Figure 8), as one would expect from the model of Weiss and Stewart (1965). Labrum and Duncan (1974) find the position shift between type III and type V sources to be predominantly outward.

A slight variation on the model of Wild and Smerd (1972) has been proposed to

1968 AUG. 21

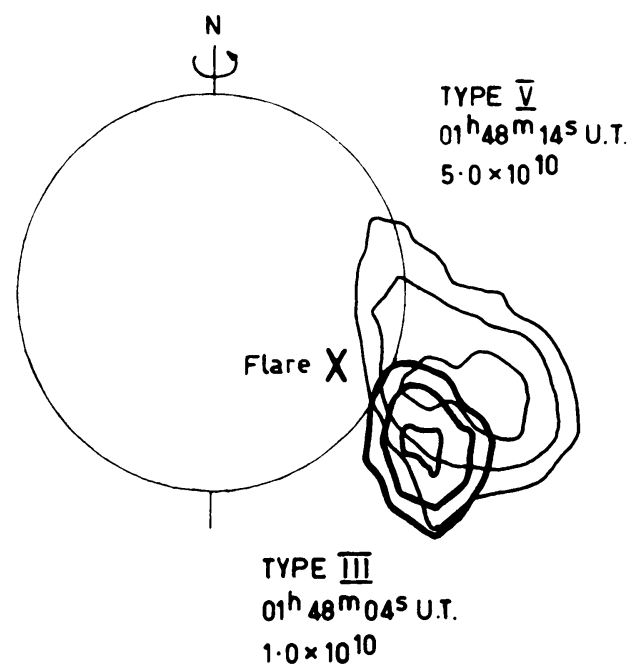

Fig. 8. $80 \mathrm{MHz}$ radioheli8graph observations showing the much larger source of a type $\mathrm{V}$ burst (light contours) displaced from that of the preceding type III burst (heavy contours). Successive contours are in the ratio $2: 1$. The numbers indicate peak beam temperature (K) (Labrum, unpublished data; Wild and Smerd, 1972).

explain the observed frequency and spatial displacements between type I and type III storm bursts (Stewart and Labrum, 1972). Unlike isolated type III bursts, the storm bursts tend to occur only at low frequencies $\leqslant 80 \mathrm{MHz}$ and are always accompanied by type I bursts at higher frequencies (Boischot $e$ t al., 1971). If the type III electrons are accelerated near the starting height of the type III burst $\left(\geqslant 0.6 R_{\odot}\right.$ above the photosphere) the acceleration region is considerably displaced, both radially and horizontally, from the type I region (Figure 9). It is proposed that an unstable region (such as the cusp of a helmet streamer (Pneuman, 1968)) is triggered by a m.h.d. disturbance from the flare region and electrons are accelerated locally and escape along the axis of the streamer.

Recently, Smith and Pneuman (1972) have cast doubts on the ability of type III electrons to escape and to be channelled along the axis of a coronal streamer. They argue that if account is taken of the finite conductivity and the azimuthal magnetic field component of a coronal streamer then the field lines do not remain open but close across the neutral sheet. Direct observations of the relative positions of type III sources and coronal streamers should settle this question. Such observations have 
been made for one type I-type III storm event by the author in collaboration with Shirley Hansen and R. Hansen and for several type III bursts by Leblanc et al. (1974). The results, shown in Figures 10a and b, (and also by Leblanc et al. (1974)) are not conclusive. There is no definite evidence for a coronal streamer near the type III

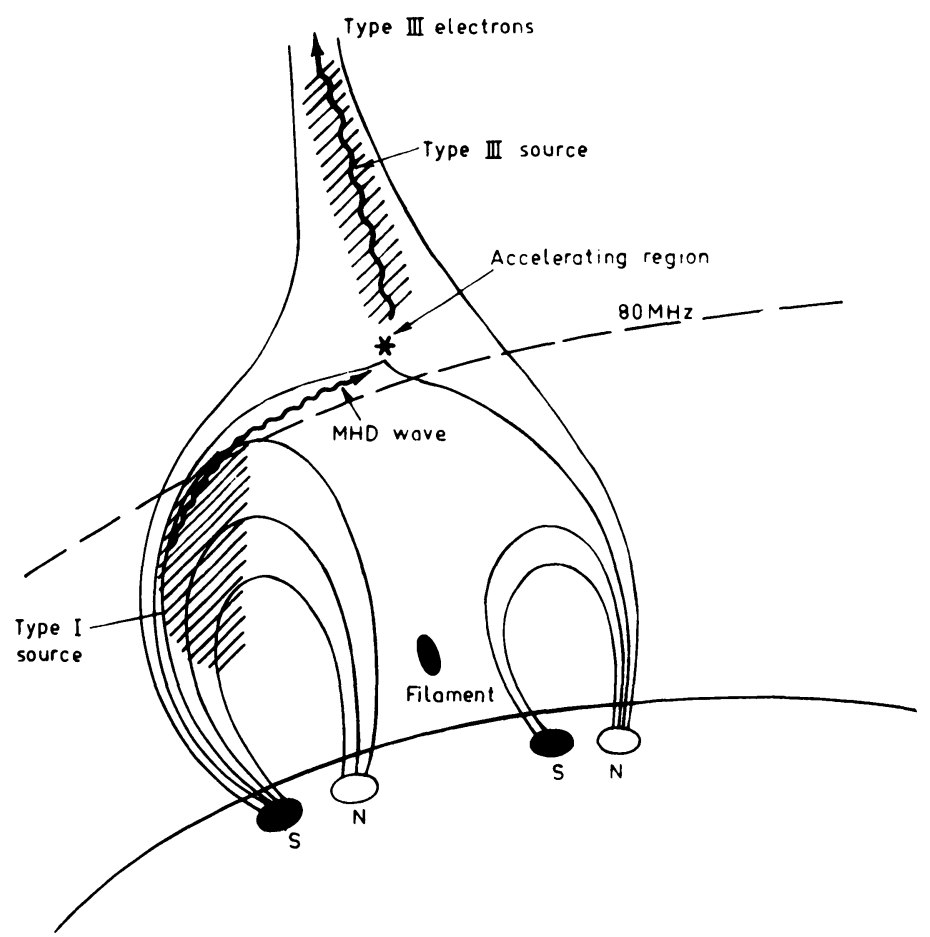

Fig. 9. Proposed model to explain the non-radial displacement between the sources of type I and type III storm bursts, observed during 1968 August 19-21 with the $80 \mathrm{MHz}$ radioheliograph (see text) (Stewart and Labrum, 1972).

position (see A of Figure 10). It may be possible to resolve this question when threedimensional density models are derived from $K$-corona and ATM-Skylab data.

\section{Propagation and Scattering of Radiation}

The propagation of type III radiation from the source to the Earth is determined by refraction by large-scale features of the solar corona, such as streamers, and by scattering on relatively small-scale irregularities. Scattering is crucial for the escape of fundamental radiation from the plasma level. Fokker (1965) showed that scattering would cause an increase in the angular size of the source and an outward shift in its apparent position. More complete studies by Steinberg et al. (1971) and Riddle (1972a) have combined the effects of refraction in a spherically symmetric corona with scattering on randomly-distributed density inhomogeneities. The results show that scattering can explain why fundamental sources are seen sometimes near the limb - 
(a) $80 \mathrm{MHz}$ POSITIONS
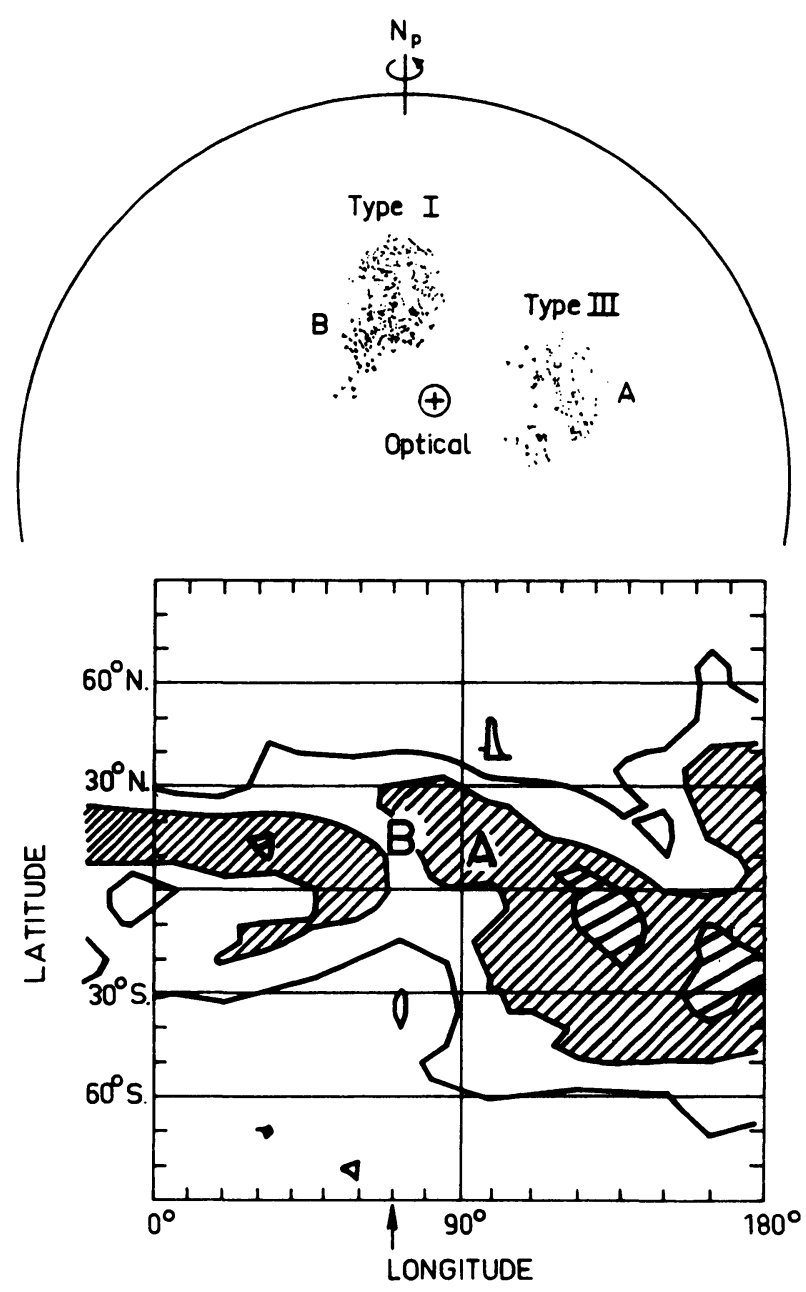

(b) $1.8 R_{\odot} I S O P H O T E S$

Fig. 10. (a) Scatter plot of centroids of all the $80 \mathrm{MHz}$ radioheliograph sources, $A$ (type III) and B (type I) for 1971 October $4-5,23^{\mathrm{h}} 10^{\mathrm{m}}$ to $01^{\mathrm{h}} 06^{\mathrm{m}}$. The cross indicates the centre of the active region McMath 11537. (b) $K$-corona isophotes (arbitrary units) derived from $1.8 R_{\odot}$ west limb scans with the coronal activity monitor on Mauna Loa, Hawaii (courtesy HAO Boulder). CMP for October 4-5 is shown by the arrow.

without scattering, the radiation is strongly beamed into the radial direction by refraction and the fundamental should only be seen near the centre of the solar disk. Riddle (1972b) has also considered a coronal streamer model. His results show that scattering can account for most of the discrepancy between electron densities derived from radio observations (Figure 2) and values obtained from optical observations (Newkirk, 1961). Weiss (1963) derived densities of $2 \times$ Newkirk's values for a coronal streamer by assuming that scattering would cause the apparent position to coincide 
with the true projected position. According to Riddle this method over-estimates densities by a factor of 1.5 (Figure 11).

Riddle (1972a, b) and Leblanc (1973) have shown that scattering also causes an increase in the angular size of the harmonic source and a position shift inward. The position shift and angular size are largest at large longitudes $\left(\geqslant 60^{\circ}\right)$ for radiation beamed into the backward direction but are considerable even for isotropic emission

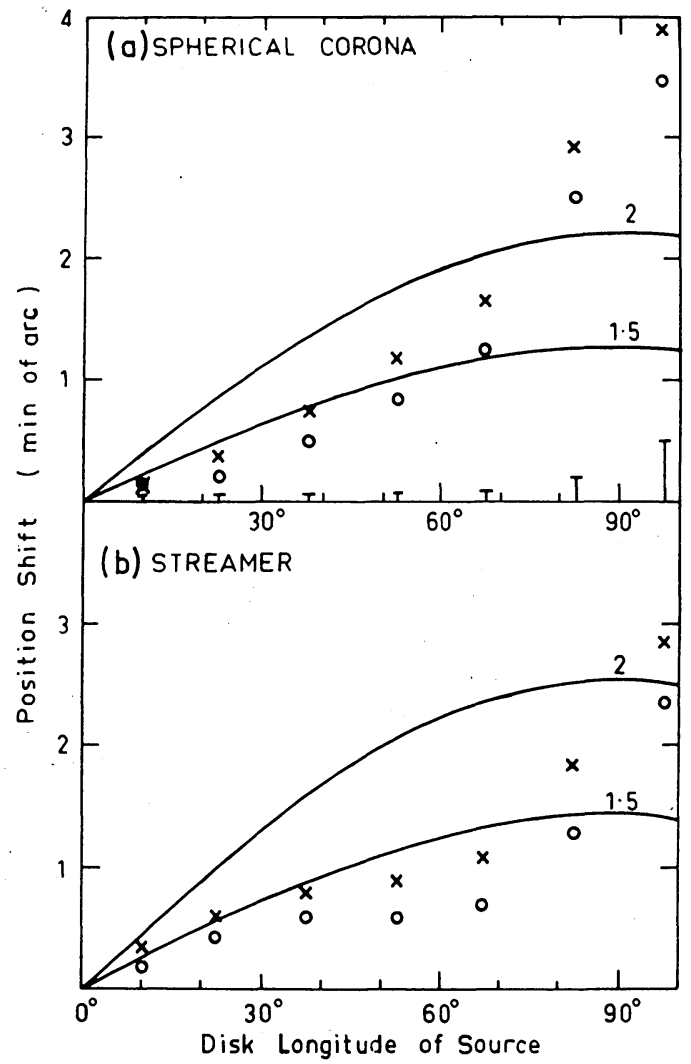

Fig. 11. Computed position shifts due to scattering and refraction of $80 \mathrm{MHz}$ radiation from a point source at the plasma level (a) in a spherically symmetric corona and (b) on the axis of a Newkirk streamer superimposed on the spherically symmetric corona model (a), with coronal temperatures of $10^{6} \mathrm{~K}$ (crosses) and $2 \times 10^{6} \mathrm{~K}$ (circles). The curves show that similar displacements can be obtained by increasing the electron density model by 1.5 and 2 times and by neglecting the effects of scattering and refraction (Riddle, 1972a).

(Figure 12b(ii) and (iv)). This result tends to weaken the argument for backward emission (Smerd et al., 1962) based on the observation that fundamental and harmonic type III bursts appear at nearly the same radial distances (Stewart, 1972; McLean, 1971, Bougeret et al., 1970). However, the position shift and angular size depend on the assumed density distributions of the large-scale as well as the small-scale features - e.g. if the source is located on the axis of a coronal streamer the position shift and 
angular size are less than in a spherically symmetrical corona (see Figure $12 \mathrm{~b}(\mathrm{i})$ and (ii)).

A study of fundamental and harmonic source sizes observed at $80 \mathrm{MHz}$ (Stewart, unpublished data) shows that: (a) the sources are usually elongated in the transverse direction; (b) the size of the harmonic source is usually equal to but sometimes larger than that of the fundamental (Figure 13); (c) the harmonic source size increases somewhat with time (Figure 14). Current scattering models may account for (c) by showing

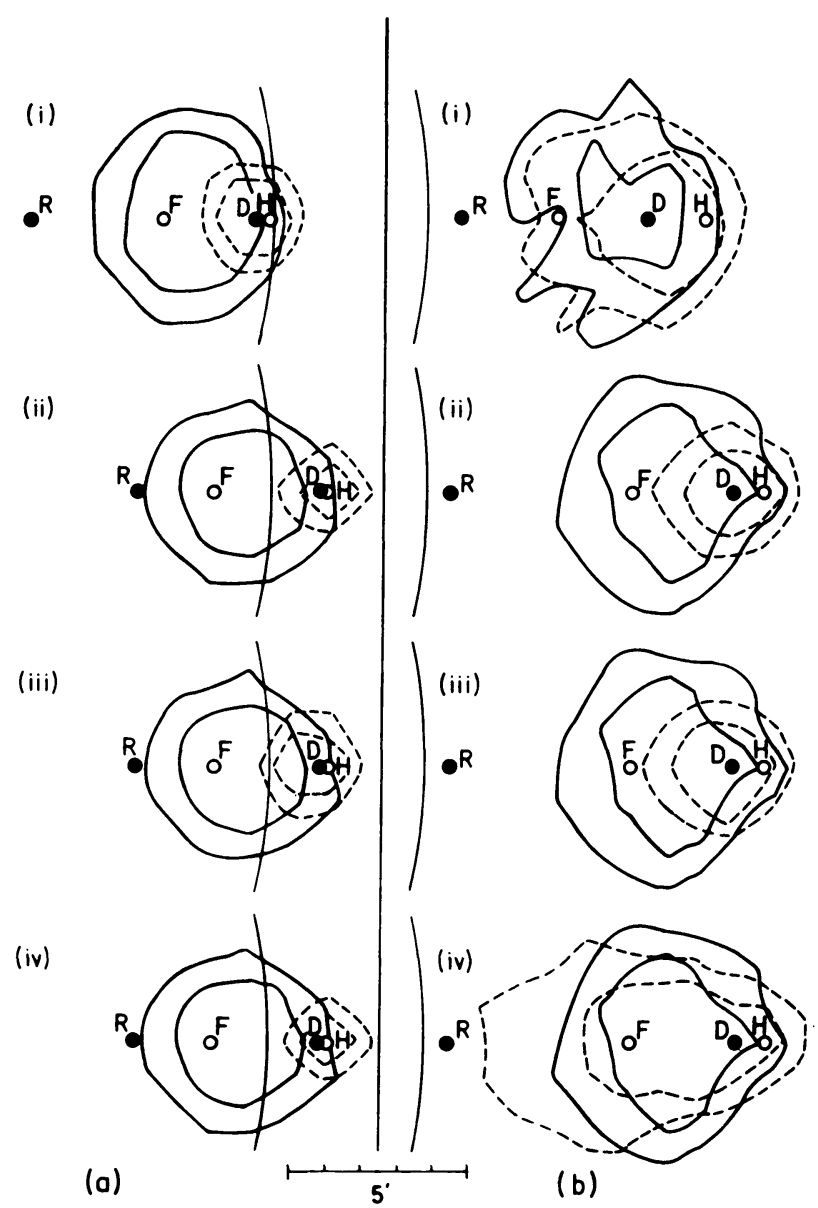

Fig. 12. Diagram showing the half-power and quarter-power contours of $80 \mathrm{MHz}$ radiation as derived from computations which include the effects of scattering and refraction. Full contours refer to radiation from an isotropically emitting 'fundamental' point source, $\mathrm{F}$, located near the $80 \mathrm{MHz}$ level. The broken contours refer to a 'harmonic' point source, $H$, located at the $40 \mathrm{MHz}$ level. The points $\mathrm{D}$ and $\mathrm{R}$ show the direction of arrival of the direct and reflected rays of the source $\mathbf{H}$ in the absence of scattering. The arc on each diagram indicates a portion of the limb of the visible disk of the Sun. The sources are located at longitude $37 \frac{1}{2}^{\circ}$ (a) and $67 \frac{1}{2}^{\circ}$ (b) in Newkirk (1961) spherically symmetric model for (i) and on the axis of Newkirk (1961) streamer model for (ii), (iii) and (iv). The source $\mathbf{H}$ is assumed to emit isotropically in (i) and (ii) and to have a radiation pattern as given by Smerd et al. (1962) in (iii) and by Zheleznyakov and Zaitsev (1970) in (iv). The exciter velocity is assumed to be one-third the speed of light (Riddle, 1972b). 
that rays scattered at large angles are more delayed than rays scattered at small angles, although the calculations have not been done as yet; they cannot account for (a) by ássuming randomly-distributed scattering centres or (b) by assuming isotropic emission. The following interpretations are worth considering: (a) the density irregularities are not randomly distributed but aligned radially like filaments; (b) the

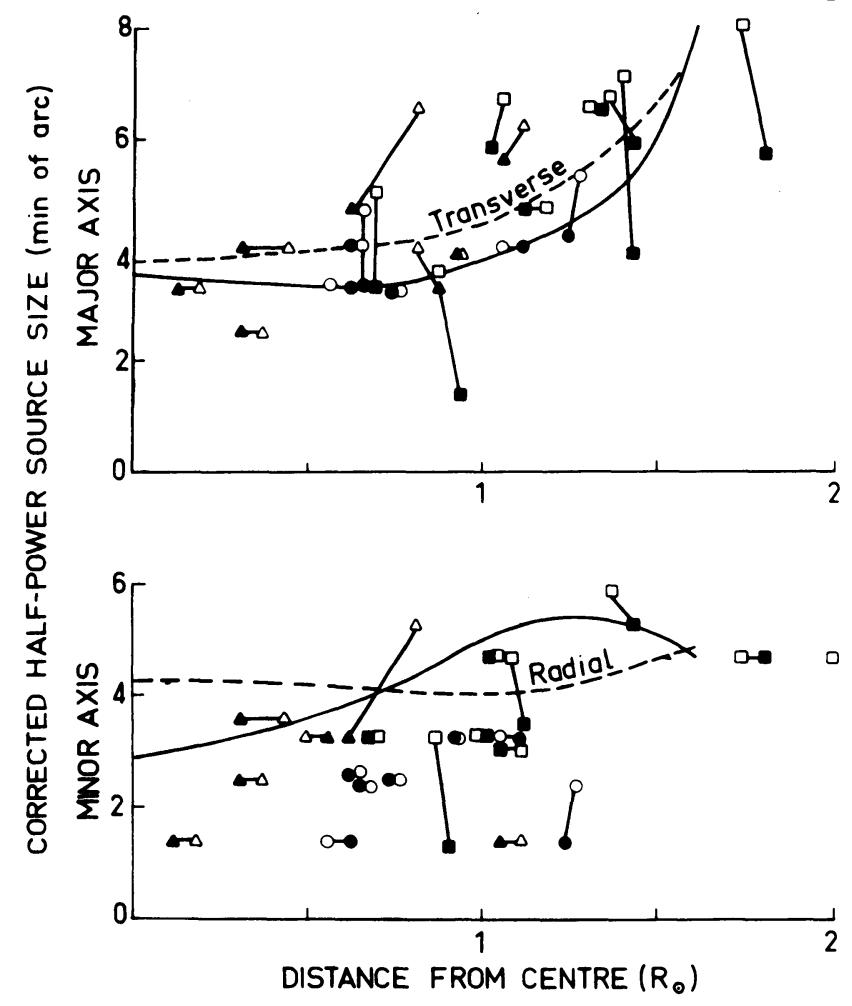

Fig. 13. Observed $80 \mathrm{MHz}$ source sizes (corrected for beam-broadening) of fundamental (filled symbols) and second-harmonic (open symbols) type III bursts. The circle indicates short-duration bursts, the square long-duration bursts, and the triangle inverted- $U$ bursts. The full and dashed curves are derived from the data of Riddle (1972a) and show the transverse (upper figure) and radial (lower figure) dimensions of the computed angular sizes for point sources in a spherically symmetric corona with randomly-distributed density irregularities. The dashed line refers to fundamental radiation from the $78 \mathrm{MHz}$ plasma level and the full line refers to harmonic radiation from the $40 \mathrm{MHz}$ plasma level. It is assumed that the sources have Gaussian brightness distributions.

emission of the harmonic is in the backward direction; (c) the intrinsic source size increases owing to stream dispersion.

Recent observations at $169 \mathrm{MHz}$ (Caroubalos and Steinberg, 1974) from the ground and from space ('Stereo-1' experiment) show that the directivity of type III bursts can be higher than the value derived by ray-tracing in a spherically symmetric corona where the scattering power is large enough to account for the observed source size. 

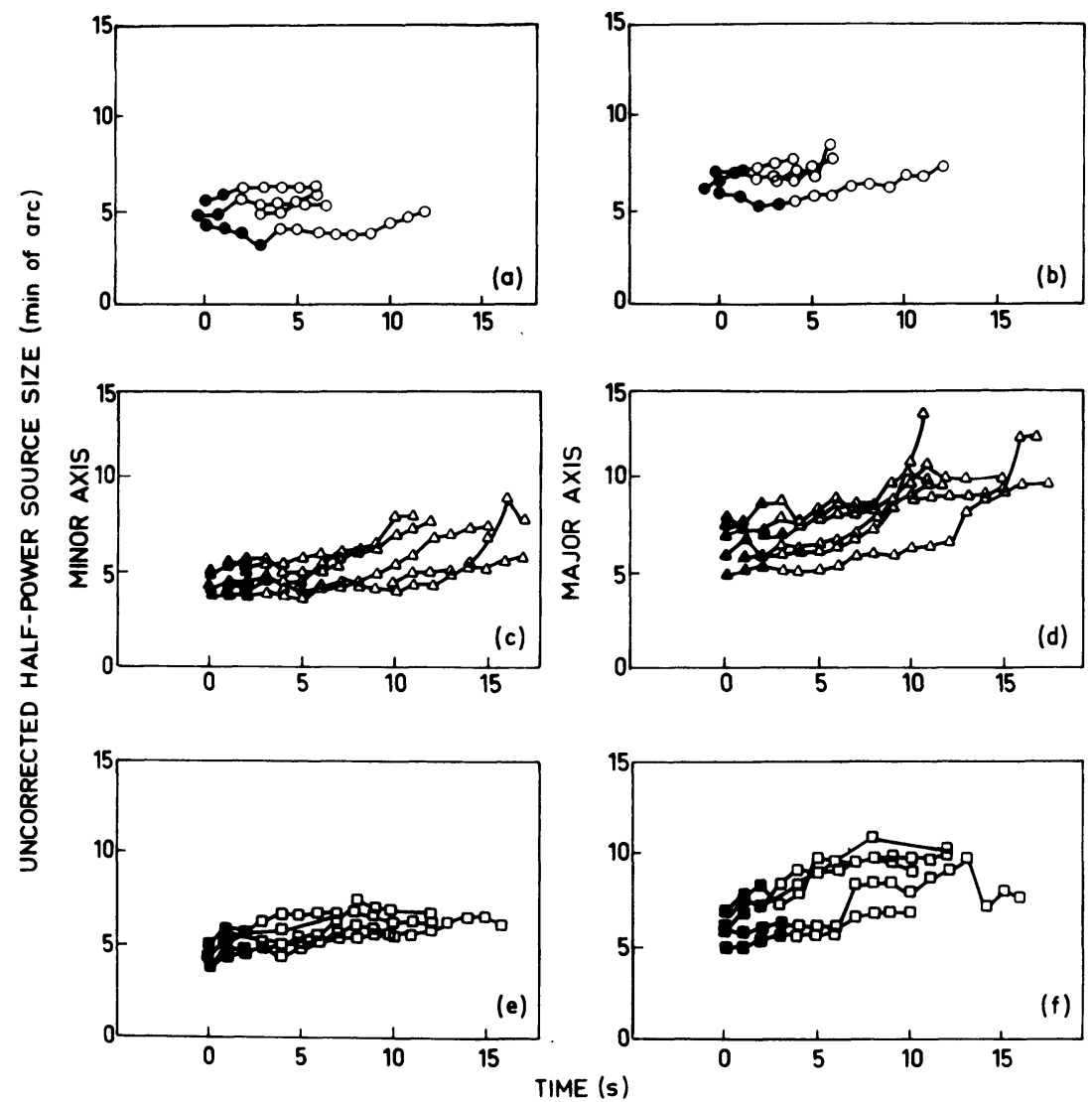

Fig. 14. Observed variation of $80 \mathrm{MHz}$ source size with time. Minor axes are shown at the left, major axes at the right. Data are from four short-duration type III bursts (a and b), five long-duration type III bursts ( $c$ and $d$ ) and five inverted- $U$ bursts (e and $f$ ). Filled symbols refer to fundamentals and open symbols to harmonics.

Large deviations from spherical symmetry and a directive harmonic source may be required to satisfy the observations. However, the 'Stereo' results are only preliminary at this stage.

\section{Linear Polarization}

Several authors (Akabane and Cohen, 1961; Bhonsle and McNarry, 1964; Daene and Voigt, 1964; Chin et al., 1971) have attempted to measure linear polarization in type III bursts by using narrow bandwidths to eliminate depolarization by Faraday rotation between the source and the observer. Their results remain controversial because no account was taken of the linear polarization caused by ground reflections.

Dodge $(1972,1973)$ has attempted to correct for ground reflections in his observations at $34 \mathrm{MHz}$. He measured a high degree of linear polarization in bursts but no Faraday rotation across the $3 \mathrm{kHz}$ bandwidth. If the result is not caused by ground reflection, then it most likely implies that the linear polarization is produced by mode- 
coupling of the radiation in regions of the corona well above the source, where the Faraday rotation is low and the effects of scattering are small.

To avoid the effects of ground reflection Grognard and McLean (1973) used a correlation technique to measure the Faraday group delay between the coherent parts of the two circular components of the radiation at $80 \mathrm{MHz}$. They found the delay was $<10 \mu \mathrm{s}$. One would expect a much higher delay from a polarized source in the corona because of differential Faraday rotation near the source.

However, Fokker (1971) has pointed out that any coherence between the two circular components of a linearly polarized signal should be washed out by scattering effects. The analysis of Steinberg et al. (1971) shows that scattered rays from a point source in the corona reach the observer with a considerable dispersion of differential time delays. The scattered rays suffer different Faraday rotations and hence even if the coherence between the two circular components were high at the source it would be considerably reduced by the time it reached the observer. Hence scattering can explain Grognard and McLean's (1973) negative result.

\section{Decay Rate and Coronal Temperature}

Many authors have derived coronal electron temperatures from the observed decay rates of the type III bursts. At a single frequency the intensity rises rapidly to a maximum then decays exponentially, as shown in Figure 15 (Aubier and Boischot, 1972). If it is assumed that the decay is due to the damping of plasma oscillations by electron-ion collisions then

$$
\tau=T^{3 / 2} / C N,
$$

where $\tau$ is the observed exponential decay constant $(s), T$ is the electron temperature $(\mathrm{K})$ and $N$ is the electron density $\left(\mathrm{cm}^{-3}\right)$; $C$ is a constant usually taken to be 40 . For emission at the fundamental plasma frequency this equation can be used to express the electron temperature in terms of the decay constant and the observing frequency, $f(\mathrm{~Hz})$ :

$$
T=0.6510^{-4} f^{4 / 3} \tau^{2 / 3}(\mathrm{~K}) .
$$

For harmonic emission the above equation over-estimates $T$ by a factor of 1.5 , because the observed frequency is twice the plasma frequency and the observed decay constant is half that of the plasma oscillations (assuming that the harmonic results from the scattering of two collisionally damped plasma waves).

Ground-based observations $(f>7 \mathrm{MHz})$ of type III bursts give values of $T$ ranging from 1 to $5 \times 10^{6} \mathrm{~K}$, if the emission is fundamental (Figure 16) and from 0.6 to $3 \times 10^{6} \mathrm{~K}$ if it is harmonic. These values are in reasonable agreement with temperatures derived from coronal emission lines (Billings, 1966). At frequencies $<7 \mathrm{MHz}$ or so the derived electron temperatures are too low and some other damping mechanisms such as Landau damping by the stream (Zaitsev et al., 1972) or the surrounding plasma (Harvey and Aubier, 1973) may apply. 


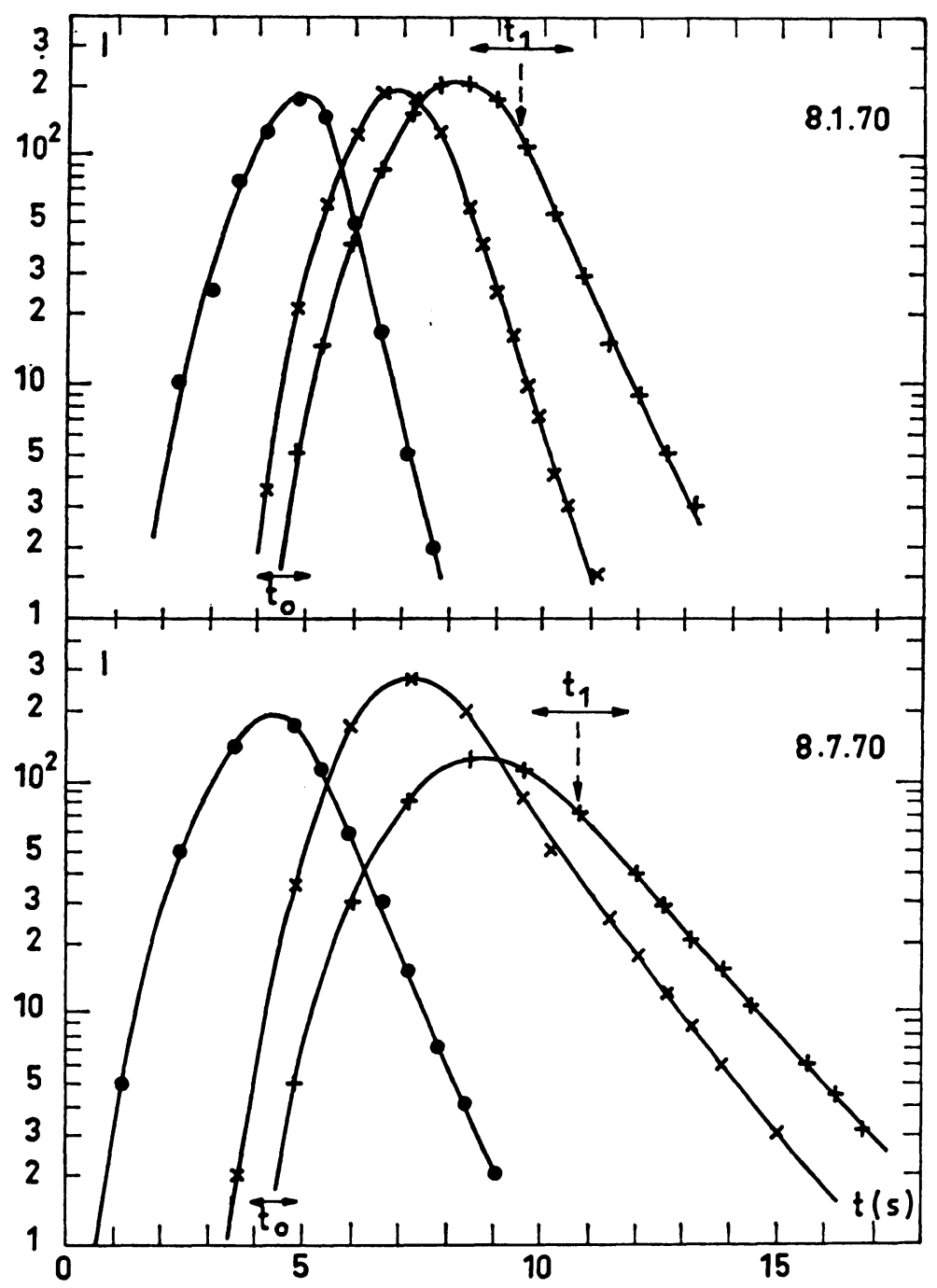

Fig. 15. Examples of type III time profiles plotted on a $\log I$-linear $t$ scale. The intensities $I$ are in arbitrary units. The symbols indicate observing frequencies as follows: $60 \mathrm{MHz} ; \times 37 \mathrm{MHz} ;+29 \mathrm{MHz}$ (Aubier and Boischot, 1972).

The work of Steinberg et al. (1971) and Riddle (1972a) suggests that scattering effect also can contribute to the observed decay rate. At $169 \mathrm{MHz}$ Steinberg et al. calculated a decay rate $\leqslant 0.2 \mathrm{~s}$ for a pulse of radiation emitted from a point source and scattered by small-scale irregularities. If interpreted in terms of collisional damping alone this corresponds to an electron temperature $\leqslant 2 \times 10^{6} \mathrm{~K}$. Hence published estimates of electron temperature based on the measured decay rates of type III bursts, ignoring scattering, can at best be taken as upper limits. Other effects, such as stream dispersion, may contribute to the initial decay rate of the type III burst (Aubier and 


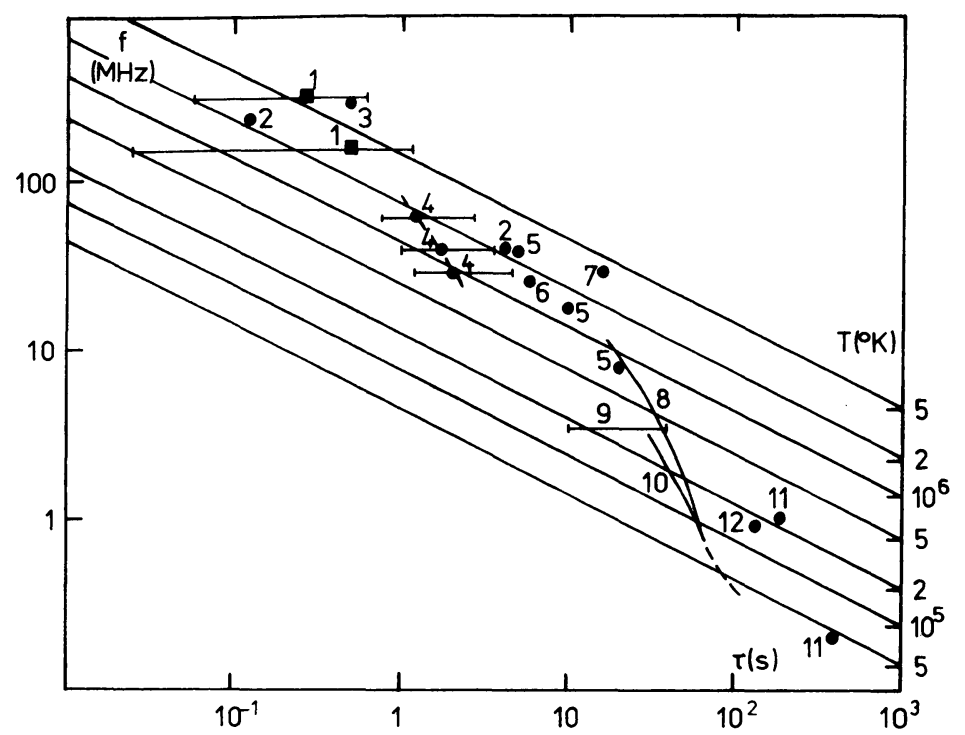

Fig. 16. Observed decay time constants, $\tau$, vs frequency. The straight lines correspond to constant temperatures computed from the equation relating decay constant to electron temperature (see text of Section 10). For references see Figure 12 of Aubier and Boischot (1972).

Boischot, 1972). Finally, it is noted that the assumption that plasma waves are damped by electron-ion collisions and not by some other mechanism is till open to question (Smith, 1974a, b).

\section{High-Resolution Spectra}

High-resolution studies of type III spectra (Elgarøy, 1961; Ellis and McCulloch, 1966, 1967; Warwick and Dulk, 1969; and de la Noë and Boischot, 1972) have shown that whilst most type III bursts have a similar appearance on both high-resolution and low-resolution spectrograms, some bursts do not. These unusual bursts often consist of narrow bands ( $\leqslant 100 \mathrm{kHz}$ ) with low drift rates. Ellis (1969) and de la Noë and Boischot (1972) have found the bands to be nearly $100 \%$ circularly polarized. Chains of bands with a leading edge drift rate similar to normal type III bursts are called type IIIb (Figure 17). These bursts are thought to be precursors of normal type III bursts (de la Noë and Boischot, 1972) but from examples studied by the author in lowresolution spectra it appears they may be the fundamental components of harmonic type III bursts.

Other high-resolution studies (De Groot, 1970; Philip, 1969) have found an apparent association between the occurrence of a type III burst at lower frequencies and pulsating quasi-periodic structures at higher frequencies. De Groot (1970) refers to associations with type I bursts, (bright spots) and broad-band structures in (type IV) continuum bursts. Philip (1969) refers to fast-drift spike bursts which may be associated with type III bursts occurring at lower frequencies. The spike bursts have very short durations, $\sim 0.1 \mathrm{~s}$ (at frequencies $\sim 200 \mathrm{MHz}$ ), relatively broad bandwidths, 


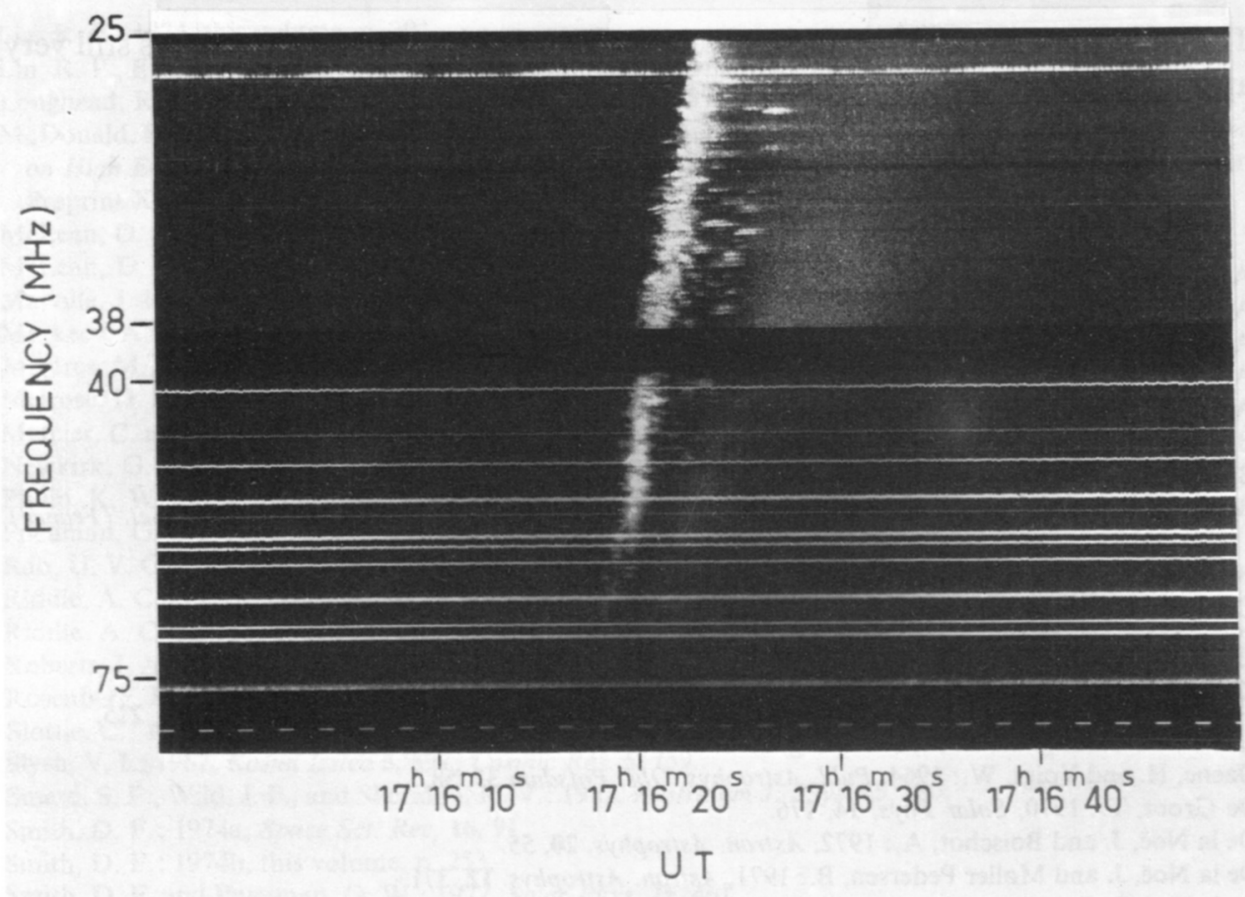

Fig. 17. Type IIIb precursor of a type III burst on 1970 August 8 observed over the 25 to $75 \mathrm{MHz}$ frequency range with a high-sensitivity spectrograph (de la Noë and Boischot, 1972).

$\leqslant 30 \mathrm{MHz}$, and are almost 100\% circularly polarized (Markeev and Chernov, 1971). We note that Fokker et al.(1974) suggest that spike bursts may be simply fine structure in a broadband continuum burst.

Another interesting relationship is that between drift pair bursts and decametre type III bursts (Roberts, 1958; de la Noë and Pederson, 1971). However, so far none of the features described above has been observed with equipment with high spatial resolution.* Until this is done interpretation based on spectral associations alone must be treated with caution.

\section{Conclusion}

Recent ground-based observations of the properties of type III bursts, particularly high-spatial resolution observations, are compatible with the earlier models of type III propagation along open field lines. The establishment of scattering as an important process in determining the position and angular size of the observed source has cast doubts on the validity of previous estimates of coronal densities and temperature.

* Recently Caroubalos et al. (1973, 1974), and Mercier and Rosenberg (1973) have combined high time resolution interferometer and spectral data in order to study source characteristics of 'paired' type III bursts and have found that the two members of the pair have sources with identical properties. 
The concept of type III propagation along the axis of a coronal streamer is still very appealing but as yet has little supporting evidence.

\section{References}

Akabane, K. and Cohen, M. H.: 1961, Astrophys. J. 133, 258.

Alvarez, H. and Haddock, F. T.: 1973, Solar Phys. 29, 197.

Anderson, K. A. and Lin, R. P.: 1966, Phys. Rev. Letters 16, 1121.

Aubier, M. and Boischot, A.: 1972, Astron. Astrophys. 19, 343.

Axisa, F., Martres, M. J., Pick, M., and Soru-Escaut, I.: 1973, Solar Phys. 29, 163.

Bhonsle, R. V. and McNarry, L. R.: 1964, Astrophys. J. 139, 1312.

Billings, D. E.: 1966, A Guide to the Solar Corona, Academic Press, N.Y.

Boischot, A., de la Noë, J., du Chaffant, M., and Rosolen, C.: 1971, Compt. Rend. Acad. Sci. (France) 272, 166.

Bougeret, J. L., Caroubalos, C., Mercier, C., and Pick, M.: 1970, Astron. Astrophys. 6, 406.

Caroubalos, C. and Steinberg, J. L.: 1974, this volume, p. 239.

Caroubalos, C., Heyvaerts, J., Pick, M., and Trottet, G.: 1974, this volume, p. 243.

Caroubalos, C., Pick, M., Rosenberg, H., and Slottje, C.: 1973, Solar Phys. 30, 473.

Chernov, G. P., Chertok, I. M., Formichev, V. V., and Markeev, A. K.: 1972, Solar Phys. $24,215$.

Chin, Y. C., Lusignan, B. B., and Fung, P. C. W.: 1971, Solar Phys. 16, 135.

Daene, H. and Voigt, W.: 1964, Publ. Astrophys. Obs. Potsdam 31, 58.

De Groot, T.: 1970, Solar Phys. 14, 176.

De la Noë, J. and Boischot, A.: 1972, Astron. Astrophys. $20,55$.

De la Noë, J. and Møller Pedersen, B.: 1971, Astron. Astrophys. 12, 371.

De la Noë, J., Boischot, A., and Aubier, M.: 1973, in R. Ramaty and R. G. Stone (eds.), Proc. Symposium on High Energy Phenomena on the Sun at Maryland, 28-30 Sept. 1972, Goddard Space Flight Center Preprint X-693-73-193, p. 602.

Dodge, J. C.: 1972, Ph. D. Thesis, University of Colorado.

Dodge, J. C. : 1973, 'Polarization Spectra of Type III Radio Bursts', paper presented at the IAU Symposium No. 57 on Coronal Disturbances, Surfers Paradise, Australia, 7-11 Sept. 1973.

Elgarøy, Ø.: 1961, Astrophys. Norv. 7, 123.

Ellis, G. R. A.: 1969, Australian J. Phys. 22, 177.

Ellis, G. R. A. and McCulloch, P. M.: 1966, Nature 211, 1070.

Ellis, G. R. A. and McCulloch, P. M.: 1967, Australian J. Phys. $20,583$.

Fainberg, J.: 1974, this volume, p. 183.

Fainberg, J. and Stone, R. G.: 1970, Solar Phys. 15, 433.

Fainberg, J., Evans, L. G., and Stone, R. G.: 1972, Science 178, 743.

Fokker, A. D.: 1965, Bull. Astron. Inst. Neth. 18, 111.

Fokker, A. D.: 1971, Solar Phys. 19, 472.

Gleeson, L.: 1965, Ph.D. Thesis, Part 1, Monash University.

Grognard, R. J. M. and McLean, D. J.: 1973, Solar Phys. $29,149$.

Haddock, F. T. and Alvarez, H.: 1973, Solar Phys. 29, 183.

Harvey, C. C. and Aubier, M. G.: 1973, Astron. Astrophys. 22, 1.

Kai, K.: 1970, Solar Phys. 11, 456.

Kane, S. R.: 1972, Solar Phys. 27, 174.

Kane, S. R.: 1974, this volume, p. 105.

Kane, S. R., Kreplin, R. W., Martres, M.-J., Pick, M., and Soru-Escaut, I. : 1974, this volume, p. 147.

Kuiper, T. B. H. and Pasachoff, J. M.: 1973, Solar Phys. 28, 187.

Kundu, M. R. : 1965, Solar Radio Astronomy, Interscience Publishers, N.Y.

Kundu, M. R., Erickson, W. C., Jackson, P. S., and Fainberg, J. : 1970, Solar Phys. 14, 394.

Labrum, N. R. and Duncan, R. A.: 1974, this volume, p. 235.

Labrum, N. R. and Stewart, R. T.: 1970, Proc. Astron. Soc. Australia 1, 316.

Leblanc, Y.: 1973, Astrophys. Letters 14, 41.

Leblanc, Y., Kuiper, T. B. H., and Hansen, S. : 1974, this volume, p. 227.

Lin, R. P.: 1970, Solar Phys. 12, 266. 
Lin. R. P.: 1974, this volume, p. 201.

Lin, R. P., Evans, L. G., and Fainberg, J.: 1973, Astrophys. Letters 14, 191.

Loughead, R. E., Roberts, J. A., and McCabe, M. K.: 1957, Australian J. Phys. 10, 483.

McDonald, F. B. and Van Hollebeke, M. A.: 1973, in R. Ramaty and R. G. Stone (eds.), Proc. Symposium on High Energy Phenomena on the Sun at Maryland, 28-30 Sept. 1972, Goddard Space Flight Center Preprint X-693-73-193, p. 404.

McLean, D. J.: 1970, Proc. Astron. Soc. Australia 1, 315.

McLean, D. J.: 1971, Australian J. Phys. 24, 201.

Malville, J. M.: 1962, Astrophys. J. 135, 834.

Markeev, A. K. and Chernov, G. P.: 1971, Soviet Astron. 14, 835.

Martres, M. J., Pick, M., Soru-Escaut, I., and Axisa, F.: 1972, Nature 236, 25.

Melrose, D. B. and Sy, W. N.: 1972, Australian J. Phys. 25, 387.

Mercier, C. and Rosenberg, H.: 1974, Solar Phys., in press.

Newkirk, G. : 1961, Astrophys. J. 133, 983.

Philip, K. W.: 1969, Bull. Am. Astron. Soc. 1, 359.

Pneuman, G. W.: 1968, Solar Phys. 3, 578.

Rao, U. V. G.: 1965, Australian J. Phys. 18, 283.

Riddle, A. C. : 1972a, Proc. Astron. Soc. Australia 2, 98.

Riddle, A. C.: 1972b, Proc. Astron. Soc. Australia 2, 148.

Roberts, J. A.: 1958, Australian J. Phys. 11, 215.

Rosenberg, H.: 1973, Ph.D. Thesis, University of Utrecht.

Slottje, C. : 1974, Astron. Astrophys. 32, 107.

Slysh, V. I.: 1967, Kosmi Issled 5, 897; Cosmic Res. 5, 759.

Smerd, S. F., Wild, J. P., and Sheridan, K. V.: 1962, Australian J. Phys. 15, 180.

Smith, D. F. : 1974a, Space Sci. Rev. 16, 91

Smith, D. F.: 1974b, this volume, p. 253.

Smith, D. F. and Pneuman, G. W.: 1972, Solar Phys. 25, 461.

Solar Radio Group Utrecht: 1974, Space Sci. Rev. 16, 45.

Steinberg, J. L., Aubier-Giraud, M., Leblanc, Y., and Boischot, A. : 1971, Astron. Astrophys. $10,362$.

Stewart, R. T.: 1965, Australian J. Phys. 18, 67.

Stewart, R. T.: 1972, Proc. Astron. Soc. Australia 2, 100.

Stewart, R. T. and Labrum, N. R.: 1972, Solar Phys. 27, 192.

Swarup, G., Stone, P. H., and Maxwell, A.: 1960, Astrophys. J. 131, 725.

Teske, R. G., Soyumer, T., and Hudson, H. S.: 1971, Astrophys. J. 165, 615.

Van Allen, J. A. and Krimigis, S. M.: 1965, J. Geophys. Res. 70, 5737.

Vorphal, J. A. and Zirin, H.: 1972, Big Bear Solar Obs. Rep. 114.

Warwick, J. W. and Dulk, G. A. : 1969, Astrophys. J. 158, L123.

Weiss, A. A.: 1963, Australian J. Phys. 16, 240.

Weiss, A. A. and Stewart, R. T.: 1965, Australian J. Phys. 18, 143.

Weiss, A. A. and Wild, J. P.: 1964, Australian J. Phys. 17, 282.

Wild, J. P.: 1950, Australian J. Sci. Res. A3, 541.

Wild, J. P.: 1970, Proc. Astron. Soc. Australia 1, 365.

Wild, J. P. and Smerd, S. F.: 1972, Ann. Rev. Astron. Astrophys. 10, 159.

Wild, J. P., Sheridan, K. V., and Neylan, A. A.: 1959, Australian J. Phys. 12, 369.

Wild, J. P., Smerd, S. F., and Weiss, A. A.: 1963, Ann. Rev. Astron. Astrophys. 1, 291.

Zaitsev, V. V., Mityakov, N. A., and Rapoport, V. O.: 1972, Solar Phys. 24, 444.

Zheleznyakov, V. V. and Zaitsev, V. V.: 1970, Soviet Astron. AJ 14, 250. 